\title{
Micorriza arbuscular em plantações de Eucalyptus cloeziana F. Muell no litoral norte da Bahia, Brasil
}

\author{
Cristiano V.M. Araújo ${ }^{1,3}$, Lander de J. Alves' ${ }^{1}$, Osvaldo M. Santos ${ }^{1}$ e Jacyr M. Alves ${ }^{2}$
}

Recebido em 14/05/2003. Aceito em 07/01/2004

\begin{abstract}
RESUMO - (Micorriza arbuscular em plantações de Eucalyptus cloeziana F. Muell no litoral norte da Bahia, Brasil). As micorrizas arbusculares são de longa data conhecidas e exploradas devido à importância ecológica e aos efeitos no crescimento e na nutrição das plantas. Eucalyptus cloeziana F. Muell, particularmente nas áreas em estudo, apresenta comportamento diferenciado quando comparado com outras espécies de eucaliptos, instalando-se em sítios de solos pobres e textura arenosa, com crescimento reduzido, dificuldades para a formação das mudas e problemas nutricionais. Objetivando avaliar a percentagem de colonização radicular e a densidade de esporos de fungos micorrízicos arbusculares em plantações de E. cloeziana, foram realizadas coletas de solo rizosférico e de raízes em 20 áreas, distribuídas em seis municípios do Estado da Bahia, Brasil. Os resultados médios da percentagem de colonização variaram de $10 \%$ a $96,66 \%$ e a densidade de esporos variou de 3 a 110 esporos $/ 50 \mathrm{~cm}^{3}$ de solo, demonstrando a grande suscetibilidade do E. cloeziana à micorrização.
\end{abstract}

Palavras-chave: Eucalyptus cloeziana, Glomales, colonização radicular, densidade de esporos

\begin{abstract}
Arbuscular mycorrhiza in Eucalyptus cloeziana F. Muell plantations in the north littoral of Bahia, Brazil). The arbuscular mycorrhizal are known and explored long ago due to the ecological significance and the effects in the growth and nutrition of the plants. Eucalyptus cloeziana F. Muell, particularly in the studied sites, exhibit differenced behaviour when compared with other eucaliptus species, establishing in sites of the poor soils and sandy texture, with reduced growth, difficulty to formation of the seedling and nutritional problems. Aiming to evaluate the percentage of mycorrhizal colonization, as well as the density of arbuscular mycorrhizal fungi spores in E. cloeziana plantations, rhizospheric soil and roots samples were collected in twenty sites, distributed in six municipalities of Bahia state, Brazil. The mean results of percentage root colonization ranged from 10 to $96.66 \%$ and spore number ranged from 3 to 110 spores $/ 50 \mathrm{~cm}^{3}$ soil, demonstrating high susceptibility of the E. cloeziana to mycorrhization.
\end{abstract}

Key words: Eucalyptus cloeziana, Glomales, root colonization, spores density

\section{Introdução}

Micorrizas arbusculares (MA) são associações mutualísticas entre fungos Glomales e raízes das plantas terrestres, nas quais as hifas dos fungos conferem superfície extra de absorção, funcionando como extensões do sistema de absorção das plantas (Went \& Stark 1968; Lopes et al. 1983). Deste modo, o fungo transfere nutrientes à planta, em especial o fósforo, e esta lhe fornece fotossintatos (Smith \& GianinazziPearson 1988). Fungos micorrízicos arbusculares (FMA) estão bem envolvidos com o estado vegetativo das plantas micotróficas (Carrenho et al. 2001a), definem seus nichos ecológicos, influenciam na composição das comunidades vegetais, na manutenção e fertilidade do solo, no estado nutricional das plantas e na ciclagem de nutrientes (Went \& Stark 1968; Harley 1989; Jeffries et al. 2003). A ocorrência dos FMA é tão ampla que mais de $80 \%$ das plantas podem formar MA (Jeffries et al. 2003), sendo considerada uma associação cosmopolita, reconhecida como parte importante e integral dos ecossistemas naturais de todo o mundo (Gadkar et al. 2001).

Algumas espécies de plantas, como o eucalipto, têm a capacidade de formar dois tipos de micorrizas, a arbuscular e a ectomicorriza (Zambolim \& Barros 1982). O estabelecimento da associação MA em eucalipto é conhecida há mais de 20 anos, e os benefícios da simbiose têm sido explorados comercialmente (St. John 1980; Zambolim \& Barros 1982; Coelho et al. 1997; Gomes \& Trufem 1998; Grazziotti et al. 1998; Santos 2001; Santos et al. 2001).

\footnotetext{
1 Universidade Federal da Bahia, Instituto de Biologia, Departamento de Botânica. Rua Barão de Geremoabo, s/n, Campus Universitário de Ondina, CEP 40170-290, Salvador, BA, Brasil (lander@ufba.br)

2 Copener Florestal Ltda, Rua CNS Junqueira, 485, CEP 48090-900, Alagoinhas, BA, Brasil

3 Autor para correspondência: cvmaraujo@hotmail.com
} 
Santos et al. (2001), em estudo realizado com cinco espécies de eucalipto, incluindo E. cloeziana, observaram uma sucessão no tipo de colonização micorrízica, sendo inicialmente dominado por FMA e posteriormente por fungos ectomicorrízicos.

O eucalipto, originário da Austrália, onde se encontram mais de 600 espécies nativas, vem sendo utilizado em programas de reflorestamento homotípico. Em diversos países essas plantações visam sua utilização em indústrias de celulose, farmacêutica e de produtos de higiene. As espécies de eucalipto apresentam características adequadas para o uso em escala comercial, tais como rápido crescimento, alta produção de celulose e resistência às adversidades das condições ambientais e às doenças (Santos et al. 2001). Por essas características, seu uso nos trópicos tem sido altamente favorável. No Brasil, a maior parcela das áreas reflorestadas com eucalipto é formada por solos de baixa fertilidade, que aliados a problemas de déficit hídrico, dificultam a produção de madeira viável economicamente (Andrade 1991 apud Marques Júnior et al. 1996).

Nas áreas estudadas, E. cloeziana vem sendo plantado desde 1992 em escala comercial e em teste de introdução de espécies, progênies e procedências, nos mais diversos tipos de solo do litoral norte da Bahia. O comportamento diferenciado de $E$. cloeziana, nesta região, há muito sugere a existência de associações mutualísticas. Logo após a germinação, ainda na fase de viveiro, aparecem enormes dificuldades para a formação das mudas, que apresentam recorrentes problemas nutricionais. $\mathrm{O}$ fornecimento pronto e repetido de nutrientes tem curto efeito, pois logo retornam os sinais de deficiência, só desaparecendo ao se ministrar no substrato das mudas doses de solo coletado de povoamentos maduros de E. cloeziana.

A dificuldade dos solos arenosos na retenção de água e de nutrientes é fator que dificulta a manutenção e o desenvolvimento da vegetação nessas áreas, sendo importante a presença de fatores, a exemplo da MA, que aumentem a capacidade de absorção de água e nutrientes pelas plantas, proporcionando sucesso no estabelecimento da vegetação (Santos et al. 1995). As MA apresentam grande importância ecológica e agronômica, porém a sua presença ou ausência pode ser influenciada por diversos fatores ambientais, tais como: condições climáticas, propriedades físicas, químicas e físico-químicas do solo, e espécie hospedeira (assim como pela idade e variedade das mesmas) (Silva et al. 2001). O conhecimento da condição micorrízica das espécies é de grande importância, pois serve de suporte para pesquisas sobre a produção de mudas e tecnologias para garantir o sucesso no reflorestamento com a espécie (Carneiro et al. 1998).

A importância estratégica de E. cloeziana para a região, pela capacidade de ocupar sítios marginais e pelas qualidades tecnológicas de sua madeira, levou à avaliação do seu status micorrízico, objetivando enriquecer as opções de manejo florestal na região.

\section{Material e métodos}

Áreas de estudo - as áreas estudadas são formadas exclusivamente por plantações de E. cloeziana utilizados comercialmente pela Copener Florestal Ltda., sendo que cada área representa um projeto, que é dividido em talhões. Estes talhões estão distribuídos pelos municípios de Alagoinhas $\left(12^{\circ} 08^{\prime} 00^{\prime \prime} \mathrm{S}\right.$ e

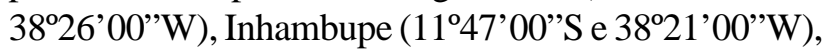

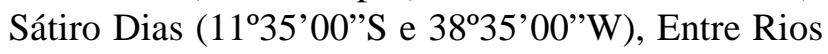

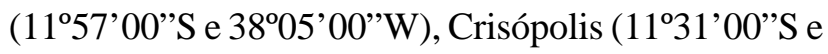

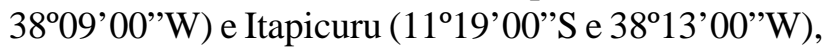
todos no Estado da Bahia, ao norte de Salvador. O relevo das regiões varia de plano a levemente ondulado. Devido à ampla disseminação do eucalipto, foi possível delimitar uma zona ótima de produção com precipitações pluviométricas médias anuais de 900 a $1.300 \mathrm{~mm}$ e sobre solos de textura leve, ainda que o horizonte arenoso se mostre bastante profundo (areias quartzosas e podzólicos de textura arenosa, arenosamédia ou média). Na Tabela 1 estão discriminadas as 20 áreas de estudo, destacando-se as práticas culturais e de manejo desenvolvidas durante a execução deste estudo, e suas principais características climáticas.

Manejo adotado nas plantações de E. cloeziana para a implantação dos projetos com E. cloeziana foi realizado manejo semelhante nas áreas. Antes do plantio retirou-se a vegetação existente, seguida de duas dragagens, sendo uma leve e uma pesada. Na sequiência, aplicou-se fosfato de Araxá (300-500kg/ha), e posteriormente foram feitos sulcos para adubação de base (6:30:6) de N-P-K. Após esse preparo realizou-se o plantio. Em seguida, fez-se capina manual e 90 dias depois aplicou-se uma última adubação, diferente em cada projeto/talhão (Tab. 1) e combateu-se as formigas com o uso de formicida. Nos anos subseqüentes foram feitos apenas controle das formigas e roçagens nas linhas (manual) e nas entrelinhas (mecanizada).

Coleta e quantificação da percentagem de colonização radicular e do número de esporos de FMA - durante o 
Tabela 1. Discriminação e caracterização das áreas de estudo utilizadas comercialmente pela Coper Florestal Ltda. (Litoral Norte da Bahia, Brasil) e dos respectivos tratos culturais e fatores climáticos.

\begin{tabular}{|c|c|c|c|c|c|c|c|c|c|c|c|c|}
\hline \multirow[t]{2}{*}{ Área } & \multirow[t]{2}{*}{ Projeto } & \multirow[t]{2}{*}{ Talhão } & \multirow[t]{2}{*}{ Município } & \multirow[t]{2}{*}{ Solo* } & \multirow{2}{*}{$\begin{array}{l}\text { Data do } \\
\text { plantio }\end{array}$} & \multirow{2}{*}{$\begin{array}{l}\text { Último } \\
\text { corte }\end{array}$} & \multirow{2}{*}{$\begin{array}{c}\text { Adubação } \\
\text { NPK }\end{array}$} & \multicolumn{3}{|c|}{ Temp. do ar $\left({ }^{\circ} \mathrm{C}\right)$} & \multicolumn{2}{|c|}{ Umidade Pluviosidade } \\
\hline & & & & & & & & Máx & Méd & Min & do $\operatorname{ar}(\%)$ & (mm/ano) \\
\hline 1 & Araticum 3 & 04 & Alagoinhas & PVd & $05 / 05 / 86$ & $30 / 10 / 95$ & 24:00:18 & 32,5 & 25,6 & 19,0 & 64,4 & 1.200 \\
\hline 2 & B \& F 1 & $05-\mathrm{A}$ & Inhambupe & $\mathrm{PCd}$ & $12 / 09 / 84$ & $12 / 09 / 94$ & $24: 00: 18$ & 32,4 & 25,3 & 17,7 & 64,1 & 900 \\
\hline 30 & Ouriçanguinhas 1 & 09 & Inhambupe & PAd & $24 / 02 / 83$ & $20 / 04 / 94$ & 24:00:18 & 32,4 & 25,1 & 18,1 & 64,7 & 900 \\
\hline 40 & Ouriçanguinhas 1 & 15 & Inhambupe & PAd & $28 / 04 / 83$ & $27 / 05 / 94$ & $24: 00: 18$ & 32,4 & 25,1 & 18,1 & 64,7 & 900 \\
\hline 50 & Ouriçanguinhas 1 & 24 & Inhambupe & PAd & $20 / 04 / 83$ & $08 / 10 / 94$ & $24: 00: 18$ & 32,4 & 25,1 & 18,1 & 64,7 & 900 \\
\hline 60 & Ouriçanguinhas 1 & $38-\mathrm{A}$ & Inhambupe & LAd & $29 / 06 / 83$ & - & 24:00:18 & 32,4 & 25,1 & 18,1 & 64,7 & 900 \\
\hline 7 & Salgado 3 & 05 & Inhambupe & PALd & $05 / 07 / 85$ & $30 / 01 / 99$ & $24: 00: 18$ & 32,4 & 25,3 & 17,7 & 64,1 & 900 \\
\hline 8 & Salgado 3 & 10 & Inhambupe & PCd & $28 / 06 / 85$ & $18 / 11 / 95$ & 24:00:18 & 32,4 & 25,3 & 17,7 & 64,1 & 900 \\
\hline 9 & Beija Flor 1 & 09 & Sátiro Dias & LVd & $15 / 06 / 83$ & $15 / 05 / 99$ & $25: 00: 25$ & 32,0 & 25,1 & 18,6 & 61,1 & 700 \\
\hline 10 & Beija Flor 1 & 11 & Sátiro Dias & LVd & $15 / 06 / 83$ & $20 / 05 / 99$ & $25: 00: 25$ & 32,0 & 25,1 & 18,6 & 61,1 & 700 \\
\hline 11 & Vitória 1 & $24-\mathrm{A}$ & Sátiro Dias & $\mathrm{AQ}$ & $15 / 07 / 82$ & $15 / 05 / 87$ & - & 32,0 & 25,1 & 18,6 & 61,1 & 700 \\
\hline 12 & Vitória 1 & 24-P & Sátiro Dias & PALd & $15 / 07 / 82$ & $15 / 05 / 87$ & - & 32,0 & 25,1 & 18,6 & 61,1 & 700 \\
\hline 13 & Nambis 1 & 08-A & Crisópolis & $\mathrm{AQ}$ & $15 / 05 / 83$ & $17 / 06 / 99$ & $10: 30: 10$ & 32,0 & 25,1 & 18,6 & 61,1 & 800 \\
\hline 14 & Nambis 1 & 08-P & Crisópolis & PVLd & $15 / 05 / 83$ & $17 / 06 / 99$ & $10: 30: 10$ & 32,0 & 25,1 & 18,6 & 61,1 & 800 \\
\hline 15 & Rio Real 3 & 06 & Itapicuru & PALd & $30 / 07 / 83$ & Cortado & $10: 30: 06$ & 32,0 & 25,1 & 18,6 & 61,1 & 800 \\
\hline 16 & Copener 1 & $19-\mathrm{A}$ & Entre Rios & PVd & $30 / 11 / 83$ & $20 / 10 / 01$ & $15: 20: 15$ & 32,5 & 25,6 & 19,0 & 64,4 & 1.200 \\
\hline 17 & Copener 1 & $19-B$ & Entre Rios & PVd & $30 / 11 / 83$ & $20 / 10 / 01$ & $15: 20: 15$ & 32,5 & 25,6 & 19,0 & 64,4 & 1.200 \\
\hline 18 & Quatis & $12-\mathrm{B}$ & Entre Rios & PAd & $19 / 07 / 96$ & - & - & 32,5 & 25,6 & 19,0 & 64,4 & 1.200 \\
\hline 19 & Copener 6 & 07 & Entre Rios & $\mathrm{Hga}$ & 09/07/96 & - & 24:00:18 & 32,5 & 25,6 & 19,0 & 64,4 & 1.200 \\
\hline 20 & Copener 6 & 07 & Entre Rios & PCd & 09/07/96 & - & $24: 00: 18$ & 32,5 & 25,6 & 19,0 & 64,4 & 1.200 \\
\hline
\end{tabular}

* (PVd - Argissolo Vermelho Distrófico; PCd - Podzólico Câmbico Distrófico; PAd - Argissolo Amarelo Distrófico; LAd - Latossolo Amarelo distrófico; PALd - Argissolo Amarelo Distrófico latossólico; LVd - Latossolo Vermelho distrófico; AQ - Areia Quartzosa; PVLd - Argissolo Vermelho Distrófico latossólico; Hga - Gleissolo Melânico Alumínico).

mês de outubro/2001 (período seco), coletaram-se três amostras simples de solo rizosférico de três espécimes de E. cloeziana, a profundidade de 5 a $20 \mathrm{~cm}$, em cada uma das áreas avaliadas. As amostras foram processadas para avaliação do $\mathrm{pH}$ de acordo com Silva et al. (1999). O teor de matéria orgânica foi determinado de acordo com Kiehl (1985).

Ainda em campo, selecionaram-se raízes finas com diâmetro igual ou menor que $2 \mathrm{~mm}$, que foram preservadas em álcool a $70 \%$ para posterior avaliação da percentagem de colonização micorrízica. Essas raízes foram inicialmente clareadas com $\mathrm{KOH} 10 \%$, mas como apresentavam taninos em excesso, o que dificultava a diafanização das mesmas, foram posteriormente tratadas com solução de $\mathrm{H}_{2} \mathrm{O}_{2}$ a $10 \%$ (Schmidt \& Scow 1986) e lavadas com água destilada. Em seguida, as raízes foram acidificadas com $\mathrm{HCl}$ a $1 \%$ e coradas com azul de algodão e lactoglicerol, de acordo com Kormanik et al. (1980). O percentual de colonização foi determinado segundo Krishna \& Bagyaraj 1984.

As amostras de solo usadas para o isolamento dos esporos de FMA foram acondicionadas conforme Trufem \& Bononi (1985) e processadas segundo Gerdemann \& Nicolson (1963), seguida de centrifugação em solução de sacarose a 50\% (Jenkins 1964). De cada área foram processadas três amostras de solo com $50 \mathrm{~cm}^{3}$ cada. Os esporos foram transferidos para placas de Petri e quantificados sob estereomicroscópio.

Análise estatística - os valores encontrados em cada réplica foram submetidos ao teste de comparação de médias (Tukey) com probabilidade de $95 \%$ e para as análises de correlação foi utilizado o teste de correlação de Spearman (Zar 1999), através do Programa GraphPad Instat versão 3.0 for Windows. Para análise de agrupamento (Valentin 2000) foi utilizando o Programa MVSP versão 3.1 for Windows, sendo utilizado o coeficiente Distância Euclidiana Simples associado ao método pela Associação Média (UPGMA). O coeficiente cofenético foi calculado utilizando o Programa FITOPAC for DOS.

\section{Resultados e discussão}

Nas áreas estudadas os valores de matéria orgânica variaram de 1,08\% (área 16) a 10,19\% (área 7) (Tab. 2). As áreas de 1 a 5 e de 8 a 20 não apresentaram diferenças significativas quanto ao 
Tabela 2. Teor de matéria orgânica e pH do solo, colonização radicular e densidade de esporos de FMA (esporos/50 $\mathrm{cm}^{3}$ de solo) em E. cloeziana F. Muell, cultivadas em 20 áreas diferentes utilizadas comercialmente pela Coper Florestal Ltda. (Litoral Norte da Bahia, Brasil).

\begin{tabular}{|c|c|c|c|c|c|c|c|}
\hline Área & Município & Projeto & Talhão & Matéria orgânica & $\mathrm{pH}$ & $\begin{array}{c}\text { Colonização radicular } \\
(\%)\end{array}$ & $\begin{array}{l}\text { Densidadede } \\
\text { de esporos }\end{array}$ \\
\hline 1 & Alagoinhas & Araticum 3 & 04 & $2,60 \mathrm{BC}$ & $4,20 \mathrm{~F}$ & $16,66 \mathrm{D}$ & $56,00 \mathrm{CD}$ \\
\hline 2 & Inhambupe & B \& F 1 & 05-A & $2,47 \mathrm{BC}$ & 4,62 CDEF & $70,83 \mathrm{ABC}$ & $81,00 \mathrm{BC}$ \\
\hline 3 & & Ouriçanguinhas 1 & 09 & $2,05 \mathrm{C}$ & $4,65 \mathrm{CDEF}$ & $10,00 \mathrm{D}$ & $12,00 \mathrm{EF}$ \\
\hline 4 & & Ouriçanguinhas 1 & 15 & $2,46 \mathrm{BC}$ & $4,91 \mathrm{ABCDEF}$ & $83,33 \mathrm{AB}$ & $12,00 \mathrm{EF}$ \\
\hline 5 & & Ouriçanguinhas 1 & 24 & $1,80 \mathrm{C}$ & $4,70 \mathrm{CDEF}$ & $16,66 \mathrm{D}$ & $11,00 \mathrm{EF}$ \\
\hline 6 & & Ouriçanguinhas 1 & $38-\mathrm{A}$ & $9,52 \mathrm{AB}$ & $5,23 \mathrm{ABCD}$ & $43,33 \mathrm{BCD}$ & $16,00 \mathrm{EF}$ \\
\hline 7 & & Salgado 3 & 05 & $10,19 \mathrm{~A}$ & $5,41 \mathrm{ABC}$ & $80,00 \mathrm{ABC}$ & $19,00 \mathrm{EF}$ \\
\hline 8 & & Salgado 3 & 10 & $1,94 \mathrm{C}$ & $4,36 \mathrm{DEF}$ & $33,33 \mathrm{CD}$ & $13,00 \mathrm{EF}$ \\
\hline 9 & Sátiro Dias & Beija Flor 1 & 09 & $3,08 \mathrm{ABC}$ & $4,68 \mathrm{CDEF}$ & $76,66 \mathrm{ABC}$ & $29,00 \mathrm{E}$ \\
\hline 10 & & Beija Flor 1 & 11 & $2,30 \mathrm{BC}$ & 5,00 ABCDEF & $42,20 \mathrm{BCD}$ & $27,00 \mathrm{EF}$ \\
\hline 11 & & Vitória 1 & $24-\mathrm{A}$ & $3,30 \mathrm{ABC}$ & $4,31 \mathrm{DEF}$ & $73,33 \mathrm{ABC}$ & $17,00 \mathrm{EF}$ \\
\hline 12 & & Vitória 1 & $24-\mathrm{P}$ & $2,67 \mathrm{BC}$ & $4,52 \mathrm{CDEF}$ & $83,33 \mathrm{AB}$ & $18,00 \mathrm{EF}$ \\
\hline 13 & Crisópolis & Nambis 1 & 08-A & $2,96 \mathrm{BC}$ & $5,43 \mathrm{ABC}$ & $83,33 \mathrm{AB}$ & $14,00 \mathrm{EF}$ \\
\hline 14 & & Nambis 1 & 08-P & $1,91 \mathrm{C}$ & $5,74 \mathrm{~A}$ & $76,66 \mathrm{ABC}$ & $27,00 \mathrm{EF}$ \\
\hline 15 & Itapicuru & Rio Real 3 & 06 & $3,64 \mathrm{ABC}$ & $4,29 \mathrm{EF}$ & $73,93 \mathrm{ABC}$ & $25,00 \mathrm{EF}$ \\
\hline 16 & Entre Rios & Copener 1 & $19-\mathrm{A}$ & $1,08 \mathrm{C}$ & $4,80 \mathrm{BCDEF}$ & $33,33 \mathrm{CD}$ & $74,00 \mathrm{BC}$ \\
\hline 17 & & Copener 1 & $19-\mathrm{B}$ & $2,24 \mathrm{C}$ & 4,53 CDEF & $16,66 \mathrm{D}$ & $35,00 \mathrm{DE}$ \\
\hline 18 & & Quatis & $12-\mathrm{B}$ & $5,30 \mathrm{ABC}$ & $4,27 \mathrm{EF}$ & $46,66 \mathrm{BCD}$ & $3,00 \mathrm{~F}$ \\
\hline 19 & & Copener 6 & $07-\mathrm{H}$ & $4,26 \mathrm{ABC}$ & $5,65 \mathrm{AB}$ & $96,66 \mathrm{~A}$ & $110,00 \mathrm{~A}$ \\
\hline 20 & & Copener 6 & 07-P & $4,65 \mathrm{ABC}$ & $5,16 \mathrm{ABCDE}$ & $86,66 \mathrm{AB}$ & $90,00 \mathrm{AB}$ \\
\hline
\end{tabular}

As médias seguidas das mesmas letras, na mesma coluna, não diferem entre si pelo teste de Tukey a 5\% de probabilidade.

percentual de matéria orgânica no solo. A matéria orgânica exerce influência sobre a estrutura e a capacidade de retenção de água do solo e sobre o perfil de nutrientes (Bagyaraj 1992). FMA podem associarse às partículas de matéria orgânica do solo utilizandoas como fonte de energia (St. John et al. 1983), podendo ainda, indiretamente, melhorar a estrutura do solo por fornecer carbono para outros microrganismos (Kabir \& Koide 2000). De acordo com Joner \& Jakobsen (1995), o desenvolvimento das hifas dos FMA pode aumentar em resposta à alta concentração da matéria orgânica, o que faz da matéria orgânica importante parâmetro nos estudos dos FMA. No entanto, são escassos os estudos sobre os efeitos da matéria orgânica na colonização radicular por FMA (Carrenho et al. 2001b).

Pela interferência da matéria orgânica na ecologia dos FMA, como já relatado, realizou-se análise de correlação com a colonização radicular, a qual demonstrou fraca correlação $(r=0,32 ; p=0,01)$. Em estudo realizado em mata de Eucaliptos, Santos (2001) observou correlação negativa $(r=-0,63)$ entre as mesmas variáveis, no período úmido, porém, no período seco, não observou correlação. As divergências quanto à relação da matéria orgânica com o desenvolvimento intra-radical dos FMA e com a densidade de esporos são grandes, podendo-se encontrar trabalhos com correlação direta (Mohammad et al. 2003) ou inversa (Carrenho et al. 2001b).

Os valores de $\mathrm{pH}$ do solo variaram de 4,20 na área 1 a 5,74 na área 14 (Tab. 2). A ocorrência e distribuição dos FMA, bem como a formação e o funcionamento da simbiose tem no $\mathrm{pH}$ um dos fatores que afetam essa associação, podendo inibir a germinação dos esporos e o crescimento de suas hifas (Siqueira et al. 1986). A ação do $\mathrm{pH}$ sobre os FMA pode ocorrer pela alteração nas propriedades químicas do substrato (atuando na solubilidade dos íons) e pela ação direta na permeabilidade das membranas do fungo (Lopes et al. 1983; Siqueira et al. 1986). Esses valores de $\mathrm{pH}$ aqui determinados, provavelmente, não influenciaram negativamente o estabelecimento da associação micorrízica, pois a literatura relata a ocorrência de FMA desde solos muito ácidos até solos com $\mathrm{pH}$ mais elevado (Entry et al. 2002).

Na análise de correlação do $\mathrm{pH}$ com a colonização radicular foi encontrada correlação positiva $(r=0,28$; $\mathrm{p}=0,03$ ), ainda menor que a encontrada com a matéria orgânica. Os resultados aqui obtidos são divergentes dos obtidos por Santos (2001), os quais apresentaram 
correlação do $\mathrm{pH}$ com a colonização radicular de $r=-0,56$ no período úmido, não obtendo correlação na época seca.

O estabelecimento de uma espécie vegetal numa comunidade depende do grau de dependência micorrízica que ela apresenta e de sua resposta fisiológica à associação micorrízica (Solís \& FerreraCerrato 1992). Zambolim et al. (1982) demonstraram que $E$. grandis e E. tereticornis inoculadas com FMA apresentaram maior crescimento que as mudas não micorrizadas, atingindo incrementos maiores que $100 \%$ em sua biomassa.

Os resultados de colonização radicular mostram percentagens variando de $10 \%$ a 96,66\% (áreas 3 e 19, respectivamente) (Tab. 2). As médias de colonização nas áreas 2, 4, 7, 9, 11, 12, 13, 14, 15, $19 \mathrm{e}$ 20 não diferiram significativamente, ocorrendo o mesmo com as áreas 1, 3, 5, 6, 8, 10, 16, 17 e 18. Em geral, quanto à colonização, não houve diferença estatística significativa entre as áreas pertencentes a um mesmo projeto, com exceção da área 4 que apresentou valor mais alto que o encontrado nas áreas 3 e 5, que fazem parte do mesmo projeto. Das 20 áreas estudadas, 11 apresentaram percentagens de colonização radicular acima de $70 \%$, demonstrando a grande capacidade de E. cloeziana em aceitar o estabelecimento da associação micorrízica. Outras cinco áreas, 6, 8, 10, 16 e 18, apresentaram um grau de colonização médio (33,33 a 46,66\%), segundo escala proposta por Carneiro et al. (1998), enquanto apenas quatro áreas, 1, 3, 5 e 17, apresentaram valores considerados baixos (10 a 16,66\%).

Em matas de eucaliptos em renovação (sete meses após o corte), no município de Entre Rios, litoral norte da Bahia, Santos (2001) encontrou valores médios de colonização de $27,77 \%$ e $58,33 \%$ nas épocas úmida e seca, respectivamente. Nas matas com indivíduos adultos (oito anos) as médias encontradas pela mesma autora foram $61,9 \%$ no período mais chuvoso e $55,55 \%$ na época seca. Zambolim \& Barros (1982) encontraram colonização em Eucalyptus spp. variando de 25 a $50 \%$, porém valores bem menores $(6,2$ a $21,7 \%)$ já foram observados em E. grandis e E. urophylla (Guimarães 1993), indicando que as percentagens de colonização radicular por FMA no gênero Eucalyptus são bastante variadas.

Durante as coletas, apenas a área 15 apresentava-se com os eucaliptos cortados, o que poderia causar uma redução na colonização radicular, em virtude do estresse sofrido e, por conseqüência, desequilíbrio na simbiose, ocasionando diminuição da percentagem de colonização. No entanto, apesar da colonização desta área ter sido alta $(73,93 \%)$, isso não significa necessariamente que não houve estresse seguido de perda de inóculo, pois a quantidade de micélio intra-radical não determina isoladamente a eficiência da simbiose, de modo que as melhores respostas de crescimento independem da percentagem de colonização do fungo (Zambolim et al. 1982).

Em geral, quantidade de arbúsculo - estrutura relacionada com a troca de nutrientes entre o fungo e a planta (Entry et al. 2002) - encontrada nas raízes foi bastante reduzida, o que pode ser indicativo de que as relações simbióticas nos talhões encontravam-se em estádios diferentes de colonização, já que estas estruturas, em alguns casos, são formadas quando novas raízes são colonizadas e, dessa forma, promovem o intercâmbio de nutrientes (Solís \& Ferrera-Cerrato 1992). Os arbúsculos têm curto período de vida, degenerando-se com 7 a 12 dias (Gadkar et al. 2001), sendo bastante sensíveis às alterações ambientais e sua presença está ligada a efeitos sazonais (St. John \& Uhl 1983). Esses fatores podem explicar a inexpressiva quantidade de arbúsculos. Por outro lado, a ausência de arbúsculos em algumas raízes pode indicar associação não-funcional ou colonização nãosimbiótica (Hirrel et al. 1978). Os resultados de Santos (2001), mesmo tendo trabalhado em duas épocas distintas, corroboram os do presente estudo, nos quais raramente os arbúsculos foram observados nas raízes de eucaliptos.

As vesículas foram observadas em muitas raízes, porém, em algumas, sua visualização foi prejudicada pela dificuldade em diafanizá-las, pois estavam bastante escurecidas devido à presença excessiva de taninos. Isto pode também ter diminuído a visualização dos arbúsculos, que são estruturas mais finas e delicadas.

Em relação à densidade de esporos de FMA foram encontrados valores médios de 3 a 110 esporos $/ 50 \mathrm{~cm}^{3}$ de solo, nas áreas 18 e 19, respectivamente. As áreas de 3 a 15 e a 17 não apresentaram diferenças significativas entre si. A segunda maior densidade de esporos de FMA foi observada na área 20, com 90 esporos $/ 50 \mathrm{~cm}^{3}$ de solo, seguida das áreas 2 e 16 . As áreas de um mesmo projeto, em geral, tiveram valores de densidade de esporos sem diferenças significativas, tendo como exceção as áreas 16 e 17, do projeto Copener, que apresentaram 74 e 35 esporos $/ 50 \mathrm{~cm}^{3} \mathrm{de}$ solo, respectivamente (Tab. 2).

Os valores de densidade de esporos, quando relacionados com a colonização radicular apresentaram coeficiente de correlação $r=0,14(p>0,05)$. Para a 
matéria orgânica e o $\mathrm{pH}$, os coeficientes de correlação com a densidade de esporos foram, respectivamente, $r=-0,08(p>0,05)$ e $r=0,16(p>0,05)$.

A área 18, apesar de baixa densidade de esporos (3 esporos $/ 50 \mathrm{~cm}^{3}$ de solo), apresentou percentagem de colonização alta $(46,66 \%)$. De acordo com Trufem \& Malatinszky (1995), o estabelecimento da associação e a colonização radicular podem ocorrer, mesmo na ausência de esporos, uma vez que outros propágulos podem estar presentes no ambiente (fragmentos de raízes colonizadas e hifas extra-radiculares que estão colonizando raízes de plantas vizinhas, por exemplo).

Adotando-se as variáveis colonização radicular e densidade de esporos de FMA, a análise de agrupamento apresentou coeficiente de correlação cofenético igual a 0,85 - valor considerado aceitável, segundo Valentin (2000). Considerando o nível de corte a Distancia Euclidiana Simples igual a 64, observa-se a formação de dois grupos, sendo um grupo composto por áreas que apresentaram percentagens de colonização e densidade de esporos elevadas (Copener 6/07-H, Copener 6/07-P e B \& F 1/05-A) e outro, representado pelas áreas restantes, com percentuais variados (Fig. 1).

Apesar de se ter trabalhado com a mesma espécie (E. cloeziana), os valores de colonização radicular e densidade de esporos foram bastante diferentes, podendo indicar que não apenas um fator está sendo responsável por tais diferenças, mas um conjunto, como por exemplo propriedades físico-químicas e biológicas do solo, disponibilidade de nutrientes no solo, fatores macro e microclimáticos, estádio de desenvolvimento e genótipo das plantas, biomassa radical, proximidade com outras espécies micotróficas, espécies de FMA

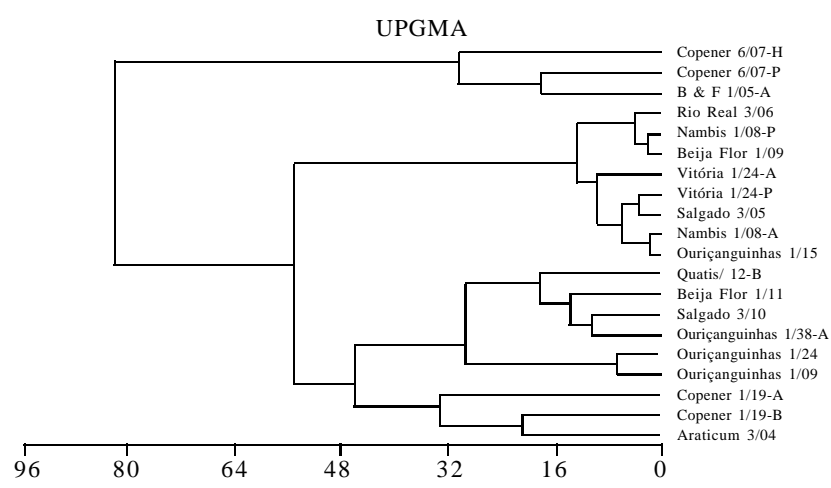

Figura 1. Dendrograma de associação das áreas utilizadas comercialmente pela Coper Florestal Ltda. (Litoral Norte da Bahia, Brasil) em relação à percentagem de colonização radicular e densidade de esporos de FMA. presentes, expansão micelial no solo, práticas culturais próprias do manejo, entre outros, que podem estar interferindo no status micorrízico do E. cloeziana.

Os resultados obtidos no presente estudo permitem-nos concluir que: E. cloeziana apresenta grande suscetibilidade à formação de micorriza arbuscular; colonização radicular, densidade de esporos, pH e matéria orgânica do solo não estão correlacionados; as práticas diferenciadas de manejo interferem na colonização radicular, porém não se pode determinar qual o fator desencadeador (ou fatores) e não se pode afirmar, utilizando apenas os dados de colonização radicular por FMA e densidade de esporos, que a dinâmica da simbiose está sendo afetada.

\section{Agradecimentos}

Os autores agradecem ao Biólogo Fabio F. Barbosa, da Universidade Federal da Bahia (UFBA) e à Dra. Rosilaine Carrenho, da Universidade Estadual de Maringá (UEM), pelas inestimáveis contribuições na construção deste trabalho.

\section{Referências}

Bagyaraj, D.J. 1992. Ecology of vesicular-arbuscular mycorrhizae. Pp. 3-34. In: D.K. Arora; B. Rai; K.G. Mukerji \& G.R. Knudsen (eds.). Handbook of Applied Mycology Soil and Plants. Banaras Hindu University, Varanasi.

Carneiro, M.A.C.; Siqueira, J.O.; Moreira, F.M.S.; Carvalho, D.; Botelho, S.A. \& Saggin-Junior, O.J. 1998. Micorriza arbuscular em espécies arbóreas e arbustivas nativas de ocorrência no sudeste do Brasil. Cerne 4(1): 129-145.

Carrenho, R.; Trufem, S.F.B. \& Bononi, V.L.R. 2001a. Fungos micorrízicos arbusculares em rizosferas de três espécies de fitobiontes instaladas em área de mata ciliar revegetada. Acta Botanica Brasilica 15(1): 115-124.

Carrenho, R.; Silva, E.S.; Trufem, S.F.B. \& Bononi, V.L.R. 2001b. Successive cultivation of maize and agricultural practices on root colonization, number of spores and species of arbuscular mycorrhizal fungi. Brazilian Journal of Microbiology 32: 262-270.

Coelho, F.B.; Borges, A.C.; Neves, J.C.L.; Barros, N.F. \& Muchovej, R.M. 1997. Caracterização e incidência de fungos micorrízicos em povoamentos de Eucalyptus grandis e Eucalyptus saligna, nos municípios de Botucatu, São José dos Campos e São Miguel Arcanjo, São Paulo. Revista Árvore 21(4): 563-573.

Entry, J.A.; Rygiewicz, P.T.; Watrud, L.S. \& Donnelly, P.K. 2002. Influence of adverse soil conditions on the formation and function of arbuscular mycorrhizas. Advances in Environmental Research 7: 123-138. 
Gadkar, V.; David-Schwartz, R.; Kunik, T. \& Kapulnik, Y. 2001. Arbuscular mycorrhizal fungal colonization. Factors involved in host recognition. Plant Physiology 127: 149-1499.

Gerdemann, J.W. \& Nicolson, T.H. 1963. Spore of mycorrhizal Endogone species extracted from soil by wet sieving and decanting. Transactions of the British Mycological Society 46(2): 235-244.

Gomes, S.P. \& Trufem, S.F.B. 1998. Fungos micorrízicos arbusculares (Glomales, Zygomycota) na Ilha dos Eucaliptos, Represa do Guarapiranga, São Paulo, SP. Acta Botanica Brasilica 12(3) (Suplemento): 393-401.

Grazziotti, P.H.; Barros, N.F.; Borges, A.C.; Neves, J.C. \& Fonseca, S. 1998. Variação sazonal da colonização de raízes de clones de híbridos de eucalipto por fungos micorrízicos no estado do Espírito Santo. Revista Brasileira de Ciência do Solo 22: 613-619.

Guimarães, L.G. 1983. Caracterização de fungos micorrízicos em povoamentos de Eucalyptus spp. em Aracruz e São Mateus, Espírito Santo e Dionísio, Minas Gerais. Dissertação de Mestrado. Universidade Federal de Viçosa, Viçosa.

Harley, J.L. 1989. The significance of mycorrhiza. Mycological Research 92: 129-139.

Hirrel, M.C.; Mehravaran, H. \& Gerdemann, J.W. 1978. Vesicular-arbuscular mycorrhizae in Chenopodiaceae and Cruciferae: do they occur? Canadian Journal of Botany 56: 2813-2817

Jeffries, P.; Gianinazzi, S.; Perotto, S.; Turnau, K. \& Barea, J.M. 2003. The contribution of arbuscular mycorrhizal fungi in sustainable maintenance of plant health and soil fertility. Biology and Fertility of Soils 37: 1-16. Jenkins, W.R. 1964. A rapid centrifugal-flotation technique for separating nematodes from soil. Plant Disease Reporter 48: 692.

Joner, E.J. \& Jakobsen, I. 1995. Growth and extracellular phosphatase activity of arbuscular mycorrhizal hiphae as influence by soil organic mater. Soil Biology Biochemistry 27(9): 1153-1159.

Kabir, Z. \& Koide, R.T. 2000. The effect of dandelion or a cover crop on mycorrhizal inoculum potential, soil aggregation and yield of maize. Agriculture, Ecosystems and Environment 78: 167-174.

Kiehl, J. 1985. Fertilizantes orgânicos. Editora Agronômica Ceres, São Paulo.

Kormanik, P.P.; Bryan, W.C. \& Schultz, R.C. 1980. Procedures and equipment for staining large number of plant roots for endomycorrhizal assay. Canadian Journal of Microbiology 26: 536-538.

Krishna, K.R. \& Bagyaraj, D.J. 1984. Growth and nutrient uptake of peanut inoculated with the mycorrhizal fungus Glomus fasciculatum compared with non-inoculated ones. Plant and Soil 77: 405-408.

Lopes, E.S.; Siqueira, J.O. \& Zambolim, L. 1983. Caracterização das micorrizas vesicular-arbusculares (MVA) e seus efeitos no crescimento das plantas. Revista Brasileira de Ciência do Solo 7: 1-19
Marques Júnior, O.G.; Andrade, H.B.\& Ramalho, M.A.P. 1996. Avaliação de procedências de Eucalyptus cloeziana F. Muell e estimação de parâmetros genéticos e fenótipos na região noroeste do estado de Minas Gerais. Cerne 2(1): 12-19.

Mohammad, M.J.; Hamad, S.R. \& Malkawi, H.I. 2003. Population of arbuscular mycorrhizal fungi in semi-arid environment of Jordan as influence by biotic and abiotic factors. Journal of Arid Environments 53: 409-417.

Santos, I.S. 2001. Fungos micorrízicos arbusculares em ambiente de mata atlântica e de Eucaliptos na região de Entre Rios, Bahia. Dissertação de Mestrado. Universidade Federal da Bahia, Salvador.

Santos, O.M.; Oliveira, N.C. \& Novais, R.F. 1995. Observações preliminares sobre fungos micorrízicos vesículo-arbusculares em plantas crescendo em dunas na Bahia. Revista Ceres 42(240): 191-202.

Santos, V.L.; Muchovej, R.M.; Borges, A.C.; Neves, J.C.L.\& Kasuya, M.C.M. 2002. Vesicular-arbuscular-ectomycorrhiza succession in seedlings of Eucalyptus spp.. Brazilian Journal of Microbiology 32: 81-86.

Schmidt, S.K. \& Scow, K.M. 1986. Mycorrhizal fungi on the Galápagos Islands. Biotropica 18(3): 236-240.

Silva, F.C.; Eira, P.A.; van Raij, B.; Silva, C.A.; Abreu, C.A.; Gianello, C.; Pérez, D.V.; Quaggio, J.A.; Tedesco, M.J.; Abreu, M.F. \& Barreto, W.O. 1999. Análises químicas para avaliação da fertilidade do solo. Pp. 75-169. In: F.C. Silva (org.). Manual de análises químicas de solos, plantas e fertilizantes. Embrapa, Brasília.

Silva, G.A.; Santos, B.A.; Alves, M.V. \& Maia, L.C. 2001. Arbuscular mycorrhiza in species of Commelinidae (Liliopsida) in the State of Pernambuco (Brazil). Acta Botanica Brasilica 15(2): 155-165.

Siqueira, J.O.; Mahmud, A.W. \& Hubbell, D.H. 1986. Comportamento diferenciado de fungos formadores de micorrizas vesicular-arbusculares em relação à acidez do solo. Revista Brasileira de Ciência do Solo 10: 11-16.

Smith, S.E. \& Gianinazzi-Pearson, V. 1988. Physiological interactions between symbionts in vesicular-arbuscular mycorrhizal plants. Annual Review of Plant Physiology and Plant Molecular Biology 39: 221-244.

Solís, M.G.R. \& Ferrera-Cerrato, R. 1992. Relación simbiótica de la micorriza vesículo-arbuscular con el estrato arbustivo e herbáceo del bosque de Zoquiapan, México. I. Época de Sequía. Revista Latino-Americana Microbiologia 34: 305-312.

St. John, T.V. 1980. Uma lista de espécies de plantas tropicais brasileiras naturalmente infectadas com micorriza vesicular-arbuscular. Acta Amazonica 10(1): 229-234.

St. John, T.V.; Coleman, D.C. \& Reid, C.P.P. 1983. Association of vesicular-arbuscular mycorrhizal hyphae with soil organic particles. Ecology 64(4): 957-958.

St. John, T.V. \& Uhl, C. 1983. Mycorrhizae in the rain forest at San Carlo de Rio Negro, Venezuela. Acta Cientifica Venezoelana 34: 233-237.

Trufem, S.F.B. \& Bononi, V.L. 1985. Micorrizas vesículoarbusculares de culturas introduzidas em áreas de cerrado. Rickia 12: 165-187. 
Trufem, S.F.B. \& Malatinszky, S.M.M. 1995. Fungos micorrízicos arbusculares de Melastomataceae e outras plantas nativas resistentes e sensíveis à poluição na Reserva Biológica do Alto da Serra de Paranapiacaba, SP, Brasil. Hoehnea 22(1/2): 77-89.

Valentin, J.L. 2000. Ecologia numérica - uma introdução à análise multivariada de dados ecológicos. Interciência, Rio de Janeiro.

Went, F.W. \& Stark, N. 1968. Mycorrhiza. Bioscience 18: 1035-1039.
Zambolim, L. \& Barros, N.F. 1982. Constatação de micorriza vesicular-aruscular em Eucalyptus spp. na região de Viçosa, MG. Revista Árvore 6(1): 95-97.

Zambolim, L.; Barros, N.F. \& Costa, L.M. 1982. Influência de micorriza do tipo vesicular-arbuscular no crescimento e absorção de nutrientes por mudas de Eucalyptus spp. Revista Árvore 6(1): 64-73.

Zar, J.H. 1999. Bioestatistical analysis. Prentice Hall, New Jersey. 